\title{
Caroline Datchary, La dispersion au travail
}

Toulouse, Octarès, 2011

\section{Sylvain Thine}

\section{(2) OpenEdition}

\section{Journals}

Édition électronique

URL : https://journals.openedition.org/travailemploi/5684

DOI : $10.4000 /$ travailemploi.5684

ISSN : 1775-416X

Éditeur

DARES - Ministère du Travail

Édition imprimée

Date de publication : 15 juin 2012

Pagination : 96-97

ISSN : 0224-4365

\section{Référence électronique}

Sylvain Thine, "Caroline Datchary, La dispersion au travail », Travail et Emploi [En ligne], 130 | avril-juin 2012, mis en ligne le 01 décembre 2012, consulté le 30 juillet 2021. URL : http://

journals.openedition.org/travailemploi/5684; DOI : https://doi.org/10.4000/travailemploi.5684

Ce document a été généré automatiquement le 30 juillet 2021.

(c) Direction de l'animation de la recherche, des études et des statistiques (Dares) 


\section{Caroline Datchary, La dispersion au travail}

Toulouse, Octarès, 2011

Sylvain Thine

\section{RÉFÉRENCE}

Caroline Datchary, La dispersion au travail, Toulouse, Octarès, 2011, 192 p.

Donner à voir, dans toutes les formes de «travaux complexes» (p.169), des situations connues et vécues telles que faire plusieurs choses à la fois, se laisser distraire, avoir des difficultés à se concentrer, tel est l'apport innovant et indéniable de l'ouvrage de Caroline Datchary. Son analyse de "la dispersion au travail », concept qu'elle a retenu pour étudier les formes de «multi-activité, polyvalence, travail d'articulation, de régulation, zapping, résistance à l'interruption » (p. 157), a l'ambition de contribuer à la description de nouvelles formes de travail. Si, comme le rappelle Laurent Thévenot dans la préface de ce livre issu d'une thèse soutenue en 2006, le capitalisme contemporain tire profit de la dispersion, les analyses fines de l'auteur montrent également que certaines catégories d'acteurs gagnent en compétences et accroissent leur satisfaction au travail grâce à leur " réactivité et leur opportunisme » face aux situations de dispersion.

2 Pour argumenter cette thèse, Caroline Datchary rappelle d'abord les conflits normatifs en matière d'attention dans l'optimisation du temps de travail. Le premier repose sur la tension entre le travail planifié et l'injonction de réactivité et d'opportunisme. Le second réside dans les normes relatives à l'attention (p.21) et notamment les pathologies telles que le trouble du déficit de l'attention avec ou sans hyperactivité (TDA/H). Après avoir évoqué la littérature consacrée à l'économie de l'attention, l'auteur précise que la dispersion peut être subie ou résulter d'une action volontaire. Puis elle analyse quatre situations de dispersion au travail en autant de chapitres. La première concerne les conducteurs de travaux d'assainissement qui travaillent dans 
des configurations très peu équipées en technologies de l'information et de la communication (TIC), où l'oral prédomine sur l'écrit. Dans ce cas, la polyvalence des tâches des conducteurs de travaux, la diversité des chantiers sur lesquels ils interviennent, et leur rythme de travail saccadé sont autant de sources de dispersion. De plus, les situations de dispersion liées aux aléas du chantier constituent autant de situations à risques et le rôle du conducteur de travaux en matière de gestion de situations de dispersion consiste en une stratégie de prévention et d'évitement des risques dans la mesure où il doit s'organiser pour ne pas mettre en péril de vies humaines, ceci d'autant que «le conducteur de travaux jouit d'un avantage de taille: les univers dans lesquels il évolue sont étanches, ce qui crée un temps interstitiel de mobilité » (p. 163). En effet, le conducteur de travaux doit faire face à des aléas climatiques et techniques (pannes ou dysfonctionnements) pour lesquels le recours aux outils technologiques n'offre pas la possibilité de différer les tâches à accomplir alors que dans les situations qui vont suivre, les acteurs sont soumis à de fortes contraintes temporelles extérieures mais ont recours aux TIC pour les aménager.

3 La seconde situation de dispersion est consacrée à des acteurs fortement équipés en TIC, évoluant dans un environnement conçu pour eux : les traders. L'image du trader entouré de multiples écrans, téléphones et pendules calées sur les horaires des plus grosses places financières mondiales représente bien son environnement informationnel complexe et multidimensionnel. Or, «cet environnement est entièrement pensé pour être au service des traders au prise avec l'évolution du marché, tant dans sa dimension spatiale (open space), qu'humaine (nombreux personnels support) ou technologique » (p. 73). Tout comme celle des conducteurs de travaux, la gestion des situations de dispersion par les traders correspond donc à une gestion frontale, organisée au cœur même de leur activité.

Le troisième cas traite d'une figure classique de la gestion des situations de dispersion: le manager. Ce cas est manifestement celui qui a été le plus approfondi, notamment par l'usage de la vidéo dont l'essentiel a pu être rapporté au lecteur par le biais de captures d'écran particulièrement significatives. L'auteur montre que, comme "l'activité du manager est souvent gouvernée par les différentes sollicitations et non par un plan d'action prédéfini », «la pression temporelle et les situations de dispersion auxquelles est confronté le manager, conduisent celui-ci à trouver en permanence un équilibre bien précaire entre la surcharge et l'optimisation de son temps » (p. 119). Il profite ainsi d'« une source de satisfaction dans le fait de combler tous les temps morts et à tirer toujours le meilleur parti de son environnement » (p. 119). La capacité du manager à se départir des situations de dispersion est déterminante pour assurer sa fonction alors même qu'il ne sera pas évalué sur ce point puisque cette compétence n'est pas formalisée dans les services des ressources humaines et encore moins scolairement certifiée. Il faut en outre souligner que les usages que fait le manager des possibilités d'automatisation offertes par les TIC sont sensiblement différents de ceux du trader dans la mesure où ses besoins ne sont pas les mêmes. Alors que le trader peut activer les fonctions de traitement en masse des informations proposées par les TIC, le manager doit essentiellement se consacrer à l'analyse de cas particuliers pour laquelle il n'y a pas de traitement automatisé possible. Le manager n'en néglige pas pour autant les TIC puisque par certains paramétrages, comme le filtrage des appels par exemple, il s'en approprie l'usage en exploitant les fonctions les plus innovantes. 
Enfin, la dernière situation de dispersion nous fait partager le quotidien fait d'urgence et de pression de deux agences d'événementiel dans lesquelles, pour les salariés, « il y a multiplicité des engagements parce que tout doit être résolu dans un temps court, orienté vers une fin - l'événement en lui-même - qui imprime à l'ensemble des activités une tension particulière " (p.124). Dans cette situation de dispersion structurelle, le salarié s'attache à rendre invisible aux yeux du client la gestion de la dispersion. La pression temporelle est collectivement prise en charge par les membres de l'équipe qui ont tout à gagner à la réussite du projet. Entre l'emballement du temps et le poids des émotions ressenties par tous, une autre dimension de la dispersion se fait jour: l'excitation. Dans cette relation de service, par nature source de dispersion en raison de sollicitations que l'on ne peut pas ignorer, une gestion des interruptions efficace est essentielle, toujours dans l'optique de gagner du temps et de limiter les risques d'erreurs. Il s'agit pour les salariés de leur donner moins de prise ou de les évacuer, notamment en adaptant leur comportement afin d'accroître leur concentration et de gagner du temps pour tenir des délais incompressibles. C'est pourquoi, adaptée au gré des situations, la stratégie des acteurs de l'événementiel comme celle des managers en matière de gestion des situations de dispersion, est, selon les cas, une stratégie de gestion frontale ou une stratégie d'évitement.

6 Considérée comme un handicap qui empêche de réaliser efficacement nos activités, la dispersion a toujours été dénoncée par l'encadrement scolaire et traquée par les éducateurs. Pourtant, comme l'auteur en fait état dans sa conclusion, les capacités et les stratégies développées pour y faire face deviennent des compétences primordiales dans l'accomplissement d'un travail afin d'assumer « une part de la charge déléguée auparavant aux compromis organisationnels» (p.5). Ainsi, les ressources technologiques ou sociales (par le recours au collectif notamment) mobilisées pour neutraliser les situations de dispersion créent des différences entre acteurs. Ces situations de dispersion sont vécues ou bien avec satisfaction ou bien comme une souffrance, ce qui peut entraîner de nombreuses conséquences physiques, psychiques et cognitives.

\section{AUTEURS}

\section{SYLVAIN THINE}

Institutions et dynamiques historiques de l'économie (IDHE) 